\title{
COMPLICACIONES QUIRÚRGICAS DE LA CIRCULACIÓN EXTRACORPÓREA
}

Ivonne Acuña*, José María Hennessey MD**, Jennifer Paola Albornoz***, Lizeth Juranny González***

\section{Resumen}

Introducción: la circulación extracorpórea en cirugía cardiovascular sustituye la función cardiaca y pulmonar, lo cual genera cambios funcionales y sistémicos que exponen al paciente a complicaciones. Objetivo: determinar las intra y posquirúrgicas en intervenidos por cirugía cardiovascular con circulación extracorpórea. Métodos: estudio descriptivo de corte transversal. Se analizaron 139 historias clínicas de adultos de enero de 2009 a junio de 2011. Las variables analizadas fueron demográficas, comorbilidades, procedimientos realizados, tiempos de bomba y de oclusión de la línea arterial, protección miocárdica utilizada y estancia hospitalaria. Las complicaciones se analizaron con el programa estadístico stata. Resultados: los sistemas con mayores complicaciones fueron cardiaco $79 \%$, circulatorio $50 \%$ y pulmonar $48 \%$. La edad promedio fue 61 años, $64 \%$ eran mujeres. Los antecedentes más frecuentes fueron $81 \%$ hipertensión arterial, 36\% tabaquismo y $35 \%$ dislipidemia. Se evidenció $14 \%$ de complicaciones intraquirúrgicas y $86 \%$ posquirúrgicas. Conclusiones: la utilización de la circulación extracorpórea conlleva mayores complicaciones posquirúrgicas. Quienes presentaron hipertensión arterial, tabaquismo y dislipidemia tuvieron mayor predisposición de complicaciones cardiacas, circulatorias y pulmonares, así como los que cursaron con tiempos prolongados de oclusión de la línea arterial y de bomba.

Palabras clave: circulación extracorpórea, perfusión, complicaciones intraquirúrgicas, complicaciones posquirúrgicas, cirugía cardiovascular.

\section{SURGICAL COMPLICATIONS OF EXTRACORPOREAL CIRCULATION}

\section{Abstract}

Introduction: Extracorporeal circulation in cardiovascular surgery replaces cardiac and pulmonary function generating functional and systemic changes which may induce complications. Objective: To determine the intra and postoperative complications in patients undergoing cardiovascular surgery with extracorporeal circulation. Methods: A cross-sectional descriptive study. We analyzed 139 clinical records of adult patients from January 2009 to June 2011. Demographic variables and other aspects as, comorbidity, procedures, pump use and arterial line partial occlusion duration, myocardial protection and hospital stay, were analyzed. Complications were analyzed using the Stata statistical software. Results: the most greatly compromised systems were the cardiac 79\%, vascular $\mathbf{5 0 \%}$ and pulmonary $48 \%$ systems. Mean age was 61 years, $64 \%$ were women. The most common antecedents were arterial hypertension $81 \%$, smoking $36 \%$ and dyslipidemia $35 \%$. We evidenced $14 \%$ of intraoperative complications and $86 \%$ of postoperative complications. Conclusions: Undergoing extracorporeal circulation leads to greater postoperative complications. Patients with past history of arterial hypertension, smoking and dyslipidemia had greater predisposition to cardiac, circulatory and pulmonary complications, as well as, those experiencing greater occlusion and pump times.

Key words: Extracorporeal circulation, perfusion, intraoperative complications, postoperative complications, cardiovascular surgery.

Fecha recibido: febrero 6 de 2015 - Fecha aceptado: julio 28 de 2015

* Exdecana Facultad de Instrumentación Quirúrgica. Profesor Titular, Fundación Universitaria de Ciencias de la Salud. Bogotá DC. Colombia.
** Cirujano cardiovascular. Instructor Asistente. Fundación Universitaria de Ciencias de la Salud. Bogotá DC.Colombia.

*** Instrumentadora Quirúrgica, Hospital de San José. Docente de práctica, Fundación Universitaria de Ciencias de la Salud. Bogotá DC. Colombia. 


\section{Introducción}

A nivel mundial algunos estudios refieren que la primera causa de muerte súbita es el infarto agudo del miocardio, lo que hace que algunos pacientes se sometan a intervenciones quirúrgicas y a procedimientos como la circulación extracorpórea ${ }^{1,2}$, la cual tiene como función mantener y controlar un adecuado flujo sanguíneo que hace indispensable sustituir las funciones cardiaca y pulmonar. Por lo anterior el paciente sufre cambios funcionales y sistémicos que lo exponen a complicaciones intra y posquirúrgicas. Estas se dan como consecuencia inherente a la complejidad de los eventos y de los métodos utilizados como la circulación extracorpórea, que permite derivar la sangre auricular a una máquina mediante un circuito en el que se mezcla con oxígeno y elimina dióxido de carbono, convirtiéndose en el método que ha permitido la universalización de la cirugía cardiaca, fundamentada en un procedimiento reproducible y seguro. ${ }^{3}$

Las principales cirugías cardiacas con circulación extracorpórea que se realizan en la institución prestadora de servicios de salud en la que se realizó este estudio son revascularización miocárdica, cambio valvular aórtico o mitral y patologías de la aorta. Los pacientes sometidos a estas cirugías pueden cursar con complicaciones cardiacas, pulmonares, renales, circulatorias, neurológicas y gastrointestinales, así como trastornos electrolíticos e infecciones en los cuales se evidencian los efectos más importantes de la circulación extracorpórea. ${ }^{4}$ Los glóbulos rojos están sujetos a daño por el paso de los rodillos de la máquina lo cual causa hemólisis; la activación de las plaquetas causa agregación y microembolismos que reducen la cantidad y funcionabilidad, produciendo anemia y trombocitopenia.,

Los pacientes sometidos a estas cirugías pueden presentar complicaciones en diferentes sistemas. A nivel cardiaco se producen por factores como hipotermia sistémica, la cardioplejia utilizada, el estado de volemia del paciente, la repercusión de la anestesia sobre el corazón y el pinzamiento en la aorta. Los trastornos más frecuentes se originan en la conducción eléctrica del corazón, las bradiarritmias y los bloqueos auriculoventriculares, en especial en los reemplazos aórtico o mitral; las arritmias se generan por trastornos electrolíticos y acidobásicos, así como la hipertensión arterial en la revascularización miocárdica. ${ }^{3,5}$

Las principales complicaciones pulmonares, como son hemotórax, neumotórax y edema agudo de pulmón, se deben a los agentes anestésicos, sedantes y analgésicos narcóticos que producen depresión ventilatoria. El largo período que permanecen los pacientes en posición supina disminuye los volúmenes pulmonares por el cierre alveolar, causando colapso pulmonar. ${ }^{3,6}$

Los trastornos de la función renal se presentan por la perfusión inadecuada y la presencia de sustancias nefrotóxicas, aunque hay otros factores que influyen como la nefropatía previa, el tiempo de oclusión de la aorta, la duración de la cirugía y el comportamiento hemodinámico llevando a una falla renal aguda. ${ }^{3,7}$ Los efectos neurológicos se clasifican en los síndromes de disfunción transitoria y de daño persistente del sistema nervioso central. ${ }^{3,8}$

Las complicaciones gastrointestinales tienen como factor etiológico una reducción de la perfusión en las vísceras abdominales dando como resultado hipoxia tisular, alteración funcional y lesión orgánica. También puede ocurrir hemorragia digestiva, infarto intestinal, perforación isquémica cecal, daño pancreático o hipoxia del hepatocito.,

Teniendo en cuenta lo anterior, este estudio pretende determinar la prevalencia de las complicaciones intra y posquirúrgicas en pacientes intervenidos por cirugía cardiovascular con circulación extracorpórea en una institución prestadora de servicios de salud, entre enero de 2009 y junio de 2011 .

\section{Metodología}

Se realizó un estudio observacional descriptivo de corte transversal. Se analizaron las historias clínicas de adultos sometidos a cirugía cardiaca con circulación extracorpórea durante enero de 2009 a junio de 2011. Se excluyeron los pacientes intervenidos por patologías congénitas, a quienes se les hubiera practicado 
por primera vez cirugía cardiaca en otra institución y las historias clínicas con datos incompletos.

Para la recolección de datos se estructuró un cuestionario de revisión de historias clínicas que incluyó variables demográficas, enfermedades concomitantes, procedimientos quirúrgicos, tiempos de permanencia en circulación extracorpórea y de oclusión de la línea arterial, protección miocárdica, complicaciones intra y posquirúrgicas, reintervenciones, ritmo cardiaco a la salida de circulación extracorpórea, derivados sanguíneos utilizados, estancia hospitalaria, permanencia en la UCI y mortalidad. La información se analizó con el programa estadístico stata 10.

Las variables cualitativas se reportan mediante frecuencias relativas y las cuantitativas con medidas de tendencia central, así como la dispersión de los datos y el análisis de correspondencias múltiples. Para determinar la relación entre las complicaciones y procedimientos quirúrgicos se realizó análisis de correspondencias múltiples en el programa spad. El estudio fue aprobado por el comité de ética en investigación con seres humanos de la FUCS y se consideró una investigación sin riesgo según la resolución 8430 de 1993.

\section{Resultados}

Se analizaron 139 historias clínicas de pacientes adultos que se sometieron a cirugía cardiaca con circulación extracorpórea. La edad promedio fue 61 años (DE 12.4). El sexo masculino predominó con $64 \%$. Las comorbilidades fueron $81 \%$ hipertensión arterial, $36 \%$ tabaquismo y 35\% dislipidemia (Tabla 1).

El reemplazo valvular se realizó en $49 \%$ de los pacientes, la revascularización coronaria en $43.8 \%$ y el remplazo valvular con revascularización miocárdica en $7.2 \%$ de los casos. Se utilizaron válvulas biológicas en $51.2 \%$ y mecánicas en $48.8 \%$, como fueron Carbomedics $39.7 \%$, St Jude $36.7 \%$ y Medtronic $17.7 \%$. Los tiempos medianos de oclusión de la línea arterial y de circulación extracorpórea en revascularización miocárdica, reemplazo valvular y revascularización cardiaca con reemplazo valvular se muestran en la Tabla 2.
Como protección miocárdica se usó custodiol en 50\%, $40 \%$ plegisol y al $10 \%$ restante se les administró solución sanguínea. Al 17\% se le aplicaron glóbulos rojos empaquetados, a $3 \%$ plaquetas y $1 \%$ plasma. A la salida de circulación extracorpórea $40.3 \%$ presentaron fibrilación, que requirió cardioversión.

Las complicaciones intraquirúrgicas fueron $14 \%$ y la más frecuente fue sangrado en 3.6\%. Las posquirúrgicas correspondieron a $86 \%$ y las más comunes fueron a nivel cardiaco la taquicardia en $27 \%$ y en las circulatorias el $33.8 \%$ desarrollaron anemia. A nivel pulmonar

\begin{tabular}{|l|c|c|}
\hline \multicolumn{3}{|c|}{ Tabla I. Características demográficas y clínicas } \\
de la poción
\end{tabular}

Tabla 2. Tiempos medianos de oclusión de línea arterial y de bomba según los procedimientos

\begin{tabular}{|l|c|c|}
\hline \multicolumn{1}{|c|}{ Procedimiento } & $\begin{array}{c}\text { Tiempo de } \\
\text { oclusión de línea, } \\
\text { minutos }\end{array}$ & $\begin{array}{c}\text { Tiempo } \\
\text { de bomba, } \\
\text { minutos }\end{array}$ \\
\hline $\begin{array}{l}\text { Revascularización } \\
\text { coronaria }\end{array}$ & 50.2 (DE:21.6) & 84.7 (DE:35.3) \\
\hline Reemplazo valvular & 73.0 (DE:26.3) & 97.6 (DE:49.6) \\
\hline $\begin{array}{l}\text { Revascularización } \\
\text { coronaria con } \\
\text { reemplazo valvular }\end{array}$ & 99.4 (DE:38.8) & 133.3 (DE:59.3) \\
\hline
\end{tabular}


$33.1 \%$ cursó con derrame pleural. En el sistema renal $21.6 \%$ manifestaron bajo gasto urinario. Los pacientes presentaron delirium en un 5\% y el $1.4 \%$ hemorragia de vías digestivas altas. El 3.6\% desarrolló sepsis e infección del sitio operatorio en la esternotomía y los trastornos electrolíticos fueron hipocalcemia en $23 \%$ e hiponatremia en $18 \%$. El $11.5 \%$ de los pacientes requirieron reintervención.

Los pacientes con complicaciones cardiacas y circulatorias se caracterizaron por ser hombres con tiempos de bomba mayor de 60 minutos y oclusión de la línea arterial por encima de 45 minutos (Figura 1). Del total de los pacientes intervenidos fallecieron 24 , lo que corresponde a $17.2 \%$, de los cuales el $6.4 \%$ fueron intraquirúrgicos y $10.8 \%$ posquirúrgicos. La permanencia promedio en la unidad de cuidado intensivo fue de 6,4 días y la estancia promedio hospitalaria de 9,7 días.

\section{Discusión}

La cirugía cardiaca muestra una respuesta inflamatoria sistémica en los pacientes intervenidos, a pesar de los avances en la tecnología y la farmacología de la perfusión, la monitorización cardiovascular así como en las técnicas anestésicas y quirúrgicas. La etiología de este fenómeno es probable que se relacione con una combinación hemodinámica peri-bypass inestable, isquemia miocárdica global, perfusión orgánica subóptima durante la circulación extracorpórea y la respuesta inmunológica del paciente, debido a la exposición al circuito extracorpóreo. Una respuesta inflamatoria balanceada y controlada puede ser potencialmente beneficiosa, ayudando al paciente a defenderse contra la infección y facilitando la cicatrización de heridas, pero la pérdida de control de esta respuesta puede conducir al inicio de una reacción inflamatoria sistémica.

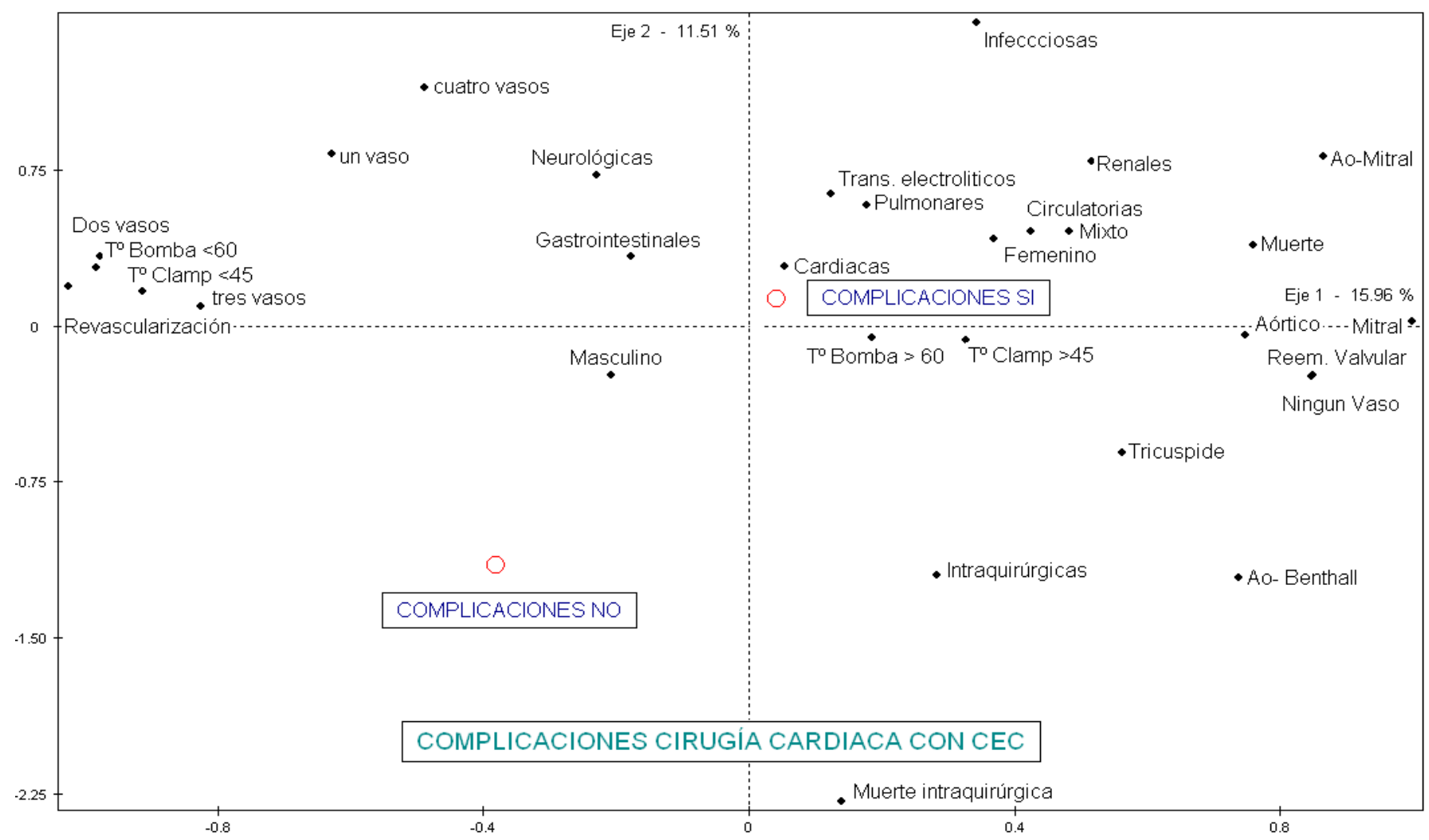

Figura I. Plano factorial. La gráfica describe la relación entre las variables consideradas en el estudio (activas) y las complicaciones (ilustrativas). La relación entre las variables se establecen por la cercania geométrica entre ellas, es decir, entre más cercanas se encuentren mayor será su relación. 
Las cirugías cardiacas con circulación extracorpórea incrementan el riesgo de presentar complicaciones al ser intervenciones muy agresivas con manipulación y colapso pulmonar durante el acto quirúrgico. ${ }^{4}$ Las complicaciones respiratorias tras la cirugía, en especial cuando se realizan bajo anestesia general, son frecuentes. En el período posoperatorio la capacidad residual funcional queda disminuida, en ocasiones hasta 70 a $75 \%$, o menos de los valores normales. Este descenso se acompaña con una pérdida de los volúmenes de reserva inspiratorio y espiratorio. Las cifras de capacidad vital forzada y del flujo espiratorio máximo manifiestan unos descensos medios del 40 al 50\% con respecto a las preoperatorias. Se reducen además la profundidad y la frecuencia de las respiraciones profundas espontáneas ("suspiros") y se altera la función ciliar. ${ }^{10}$

El porcentaje de complicaciones respiratorias presentadas fue de $48 \%$ lo que comparado con la literatura resulta inferior a lo obtenido por Hansen ${ }^{11}$ (75\%), Gunnarsson $^{10}(86.6 \%)$ y Wiren ${ }^{6}(54 \%)$. Esta incidencia depende además del tipo de cirugía y de la situación preoperatoria del paciente, y de los cuidados perioperatorios. De las complicaciones presentadas, el número de pacientes con atelectasias fue $25.5 \%$, inferior al descrito en los trabajos del grupo de Hedenstierna ${ }^{10}$ sobre una población de más de 7000 (80\%). Mustafá $\mathrm{y} \mathrm{col}^{12}$ encontraron una incidencia de derrames pleurales próximos a $12 \%$, inferiores a este estudio con un $33.1 \%$.

Durante muchos años se consideró la circulación extracorpórea como la principal y única responsable de la disfunción y la insuficiencia renal aguda en los pacientes cardiacos sometidos a cirugía con bomba, pero existe evidencia que ante una función renal preoperatoria normal no hay daño significativo. ${ }^{7}$ Estos hallazgos han permitido desmitificar los temores de las técnicas quirúrgicas con bomba; por otra parte, los estudios han enfatizado el rol del perioperatorio como principal período en el cual se producen este tipo de daños. ${ }^{8}$ Hoy se acepta en la literatura que el principal factor de daño renal posoperatorio es la presencia de disfunción renal preoperatoria: valores sobre 1.5 o $2.0 \mathrm{mg} / \mathrm{dl}$ de creatinina plasmática parecen ser críticos. ${ }^{7,13,14}$ Los pacientes con patología valvular han sido también considerados dentro del grupo de alto riesgo de presentar daño renal. En la institución de este estudio se demostró que $21.6 \%$ manifestaron bajo gasto urinario y falla renal $5 \%$.

Se ha descrito disfunción poscirugia cardiaca en todos los niveles del sistema nervioso. Las complicaciones se pueden clasificar en tres: 1) accidentes vasculares encefálicos con una incidencia de 1 a $5 \%$; 2) encefalopatías 3 a $15 \%$ y 3) alteraciones de las pruebas neuropsicológicas en 30 a $80 \%$. Los primeros suelen ser isquémicos o hemorrágicos que son excepcionales; se atribuyen a una híperperfusión en pacientes con alteración de la autorregulación cerebral por hipoperfusión crónica y pueden comprometer el territorio de una o más arterias o bien el área limítrofe. Ambos cursan con déficit neurológico focal. ${ }^{8}$ Las embolias son el mecanismo más propuesto; así las microembolias serían el sustrato del delirium y las alteraciones de las pruebas neuropsicológicas se les atribuye el sopor, coma y accidentes vasculares encefálicos de territorio limítrofe. De los 139 pacientes estudiados, tres presentaron accidente cerebrovascular $(2.2 \%)$ y siete delirium (5\%), valores acordes con los estudios a nivel mundial. ${ }^{8}$

La identificación de complicaciones gastrointestinales después de una cirugía cardiaca puede ser difícil debido a que los pacientes permanecen sedados y con ventilación mecánica durante horas o días; además la mayoría presenta diferentes grados de íleo paralítico. Todos estos factores enmascaran los síntomas y se perturba el diagnóstico precoz de las complicaciones por lo que pueden pasar inadvertidas y se diagnostiquen post mórtem. ${ }^{15,16} \mathrm{Al}$ igual que en otros estudios la gastroenteropatía aguda hemorrágica fue la complicación más frecuente y apareció a pesar del uso de fármacos protectores de la mucosa gástrica. ${ }^{15,16}$

La pancreatitis es también infrecuente. ${ }^{17}$ La hipercalcemia se considera una causa, aunque el mecanismo exacto se desconoce. Es por eso que algunos autores plantean la hiperamilasemia como un indicador de pancreatitis subclínica. La causa principal puede ser la disminución de la excreción renal más que el daño celular. ${ }^{18}$ La reducción del flujo sanguíneo esplácnico es el factor fundamental de la mayoría de las compli- 
caciones gastrointestinales, a la que contribuyen la vasoconstricción y el bajo gasto cardiaco que suele agravar la hipoperfusión por la necesidad de vasopresores y el uso de la circulación extracorpórea; además es posible que la isquemia mesentérica se agrave por aterosclerosis preexistente. ${ }^{15}$

En el sistema gastrointestinal 1.4\% manifestaron hemorragia de vías digestivas, ubicándose por debajo del rango reportado a nivel mundial que es de 3\%; 16 pacientes fueron reintervenidos por sangrado lo que corresponde a $11.5 \%$. Estudios amplios y a nivel mundial reportan que ocurre en 5 a $7 \%$ de los casos 9 , encontrando entre nosotros una incidencia por encima de lo esperado. Un estudio realizado en la Fundación Cardioinfantil Santafé de Bogotá en 2005 con 536 pacientes $^{19}$, reporta una incidencia de $9 \%$. El riesgo de sangrado parece que tiene una relación directa con la edad, en especial en la población mayor de 70 años.

\section{Conclusiones}

La prevalencia de las complicaciones en pacientes intervenidos por cirugía cardiovascular con circulación extracorpórea en una institución prestadora de servicios de salud de Bogotá DC., Colombia, fue de $14 \%$ intraquirúrgico y $86 \%$ posquirúrgico. La enfermedad cardiovascular afecta más al sexo femenino. Los pacientes con antecedentes de hipertensión arterial, tabaquismo y dislipidemia cursaron con más complicaciones en los sistemas cardiaco, circulatorio y pulmonar, así como los que tuvieron tiempos de oclusión de la línea arterial y bomba prolongados. Como complemento de este estudio se hace necesario incluir otros centros de estudio para determinar las complicaciones presentadas y así poder llevar un registro que tienda a disminuirlas en el tratamiento quirúrgico.

\section{Referencias}

1. Lahoz C, Mostaza JM. La aterosclerosis como enfermedad sistémica. Revista Española de Cardiología. 2007;60(02):184-95.

2. Jaramillo Gomez NI, Torres de Galvis Y. Aspectos epidemiológicos de la enfermedad cardiovascular: factores de riesgo a la luz de Framingham2004; 18(2):[4568 pp.]. Available from: http://pdf.revespcardiol.org/watermark/ctl servlet? $\mathrm{f}=10 \&$ pident_articulo=13073893\&pident_usuario=0\&pcontactid=\&pident_revi sta $=25 \&$ ty $=88 \&$ accion $=$ L \&origen $=$ cardio $\& w e b=w w w . r e v e s p c a r d i o l . o r g \& l a n=e$ s\&fichero $=25$ v58n04a13073893pdf001.pdf.
3. Gomar Sancho C, Pomar José Luis Mata, Ma Teresa, Asociación Española de Perfusionistas. Cambios fisiopatologicos producidos por la circulación extracoropórea y sus consecuencias clinicas en el adulto y el niño. Fisiopatología y técnicas de circulación extracorpórea. [Barcelona]: Ergón; 2003. p. 367-430.

4. Anthony A, Sendelbach S. Postoperative complications of coronary artery bypass grafting surgery. Critical care nursing clinics of North America. 2007;19(4):40315, vi. Epub 2007/11/21.

5. Affronti A, Gomar Ce, Mata MTeP, José Luis[ed.]; , García Asenjo Mp. Técnica y clínica de la circulación extracorporea en adultos y niños. Fisiopatología y técnicas de circulación extracorpórea. 2a. ed ed. [Barcelona]: Ergón; 2012. p. 183-357.

6. Wiren JE, Janzon L. Respiratory complications following surgery. Improved prediction with preoperative spirometry. Acta anaesthesiologica Scandinavica. 1983;27(6):476-9. Epub 1983/12/01

7. Lema G, Meneses G, Urzua J, Jalil R, Canessa R, Moran S, et al. Effects of extracorporeal circulation on renal function in coronary surgical patients. Anesthesia and analgesia. 1995;81(3):446-51. Epub 1995/09/01

8. Breuer AC, Furlan AJ, Hanson MR, Lederman RJ, Loop FD, Cosgrove DM, et al. Central nervous system complications of coronary artery bypass graft surgery: prospective analysis of 421 patients. Stroke; a journal of cerebral circulation. 1983;14(5):682-7. Epub 1983/09/01

9. Poirier B, Baillot R, Bauset R, Dagenais F, Mathieu P, Simard S, et al. [Abdominal complications associated with cardiac surgery. Review of a contemporary surgical experience and of a series done without extracorporeal circulation]. Canadian journal of surgery Journal canadien de chirurgie. 2003;46(3):176-82. Epub 2003/06/19. Les complications abdominales associees a la chirurgie cardiaque: a propos d'une experience chirurgicale contemporaine et examen d'une population operee sans circulation extracorporelle.

10. Gunnarsson L, Tokics L, Gustavsson H, Hedenstierna G. Influence of age on atelectasis formation and gas exchange impairment during general anaesthesia British journal of anaesthesia. 1991;66(4):423-32. Epub 1991/04/01.

11. Hansen G, Drablos PA, Steinert R. Pulmonary complications, ventilation and blood gases after upper abdominal surgery. Acta anaesthesiologica Scandinavica. 1977;21(3):211-5. Epub 1977/01/01

12. Mustafa KY, Nour MM, Shuhaiber H, Yousof AM. Pulmonary function before and sequentially after valve replacement surgery with correlation to preoperative hemodynamic data. The American review of respiratory disease. 1984;130(3):400-6. Epub 1984/09/01.

13. Chertow GM, Burdick E, Honour M, Bonventre JV, Bates DW. Acute kidney injury, mortality, length of stay, and costs in hospitalized patients. Journal of the American Society of Nephrology : JASN. 2005;16(11):3365-70. Epub $2005 / 09 / 24$

14. Urzua J, Troncoso S, Bugedo G, Canessa R, Munoz H, Lema G, et al. Renal function and cardiopulmonary bypass: effect of perfusion pressure. Journal of cardiothoracic and vascular anesthesia. 1992;6(3):299-303. Epub 1992/06/01.

15. Ghosh S, Roberts N, Firmin RK, Jameson J, Spyt TJ. Risk factors for intestinal ischaemia in cardiac surgical patients. European journal of cardio-thoracic surgery : official journal of the European Association for Cardio-thoracic Surgery. 2002;21(3):411-6. Epub 2002/03/13.

16. Kavarana MN, Frumento RJ, Hirsch AL, Oz MC, Lee DC, Bennett-Guerrero E Gastric hypercarbia and adverse outcome after cardiac surgery. Intensive care medicine. 2003;29(5):742-8. Epub 2003/04/12.

17. Stefaniak T, Glowacki J, Dymecki D, Lachinski A, Gruca Z. Pancreatitis following heart transplantation: report of a case. Surgery today. 2003;33(9):693-7. Epub 2003/08/21

18. Ihaya A, Muraoka R, Chiba Y, Kimura T, Uesaka T, Morioka K, et al. Hyperamylasemia and subclinical pancreatitis after cardiac surgery. World journal of surgery. 2001;25(7):862-4. Epub 2001/09/27

19. Dussich A, Vanegas MV. Factores predictores de sangrado mediastinal no quirúrgico en pacientes adultos sometidos a cirugía cardiaca. Revista Ciencias de la Salud. 2005;3(1):25-36 